\title{
Dolo eventual en la conducción temeraria de automotores
}

\section{Gross negligence in the reckless driving of motor vehicles}

\author{
Italo Dalton Gaibor Yánez
}

Magíster en Derecho Procesal

Universidad Estatal de Bolívar, Guaranda - Ecuador

Correo electrónico: igaibor@ueb.edu.ec

https://orcid.org/0000-0002-8989-0227

\author{
Diego Mauricio Bonilla Jurado \\ Magíster en Gestión de Proyectos \\ Universidad Tecnológica Indoamerica - Ecuador \\ Correo electrónico: diegobonilla@uti.edu.ec \\ https://orcid.org/0000-0002-4784-7861
}

\section{Resumen}

El dolo constituye una figura de difícil determinación dentro del derecho penal, de manera especial si se pretende valorar la intención que tiene un individuo al momento de cometer un delito. Bajo este contexto, el objetivo del presente manuscrito se centra en recopilar información acerca del dolo eventual, la conducción temeraria y si esto se encuentra contemplado dentro de la legislación ecuatoriana. Para esto fue necesario acudir a fuentes de información secundaria

Cómo citar este artículo:

Gaibor, I. D. \& Bonilla, D. M. (2020). Dolo eventual en la conducción temeraria de automotores. Revista de la Facultad de Derecho y Ciencias Políticas, 50 (133), pp. 243-258.

doi: http://dx.doi.org/10.18566/rfdcp. v50n133.a01

Recibido: 10 de octubre de 2019.

Aprobado: 03 de marzo de 2020. 
como libros, códigos, leyes, artículos, entre otros, para determinar o no su aplicación dentro del contexto nacional. Con estos antecedentes se puede concluir que el estudio pone de manifiesto las falencias existentes dentro del marco legal del país, tanto con la Ley Orgánica de Transporte Terrestre y Seguridad Vial cuya última modificación corresponde al 2014 y el Código Orgánico Integral Penal (2014). En ninguna de estas dos normativas existe algún artículo que castigue la conducción temeraria fundamentado en el dolo eventual.

\section{Palabras clave}

Accidentes de tránsito, conducción temeraria, Código Orgánico Integral Penal (COIP), dolo eventual, Ley Orgánica de Transporte Terrestre y Seguridad Vial.

\section{Abstract}

The intent constitutes a hard to determine figure within criminal law, especially if it is intended to assess the intention of an individual at the time of committing a crime. Within this context, the objective of this paper is centered around gathering information about gross negligence, reckless driving, and if this is contemplated in Ecuadorian Legislation. To this end, it was necessary to resort to secondary information sources such as books, codes, laws, and articles, among others, to determine or not their application within the national context. Considering this background, it is possible to conclude that the study highlights the shortcomings of the country's legal framework, both with the Ley Orgánica de Transporte Terrestre y Seguridad Vial whose last amendment corresponds to 2014 and the Código Orgánico Integral Penal (2014). In none of these regulations is there an article that punishes reckless driving based on gross negligence.

\section{Keywords}

Traffic accidents, reckless driving, Código Orgánico Integral Penal (COIP), gross negligence, Ley Orgánica de Transporte Terrestre y Seguridad Vial.

\section{Introducción}

Durante los últimos años, el problema de seguridad vial y sus consecuencias ha cobrado gran importancia en el contexto mundial, dado que el número de muertos por accidentes de tránsito se aproxima a los 1,2 millones de personas por año. Esto representa más de $2,1 \%$ de la tasa de mortalidad mundial (Organización Mundial de la Salud, 2014). En el contexto ecuatoriano, de igual manera los accidentes de tránsito hoy en día representan un serio problema 
social que involucra a un sinnúmero de conductores y peatones. Según las estadísticas de la Agencia Nacional de Tránsito (2019) durante el último año se han registrado un total de 24.595 casos asociados a diferentes tipos de siniestros en carretera (arrollamientos, atropellos, caídas de pasajeros, choque frontal, choque lateral, choque posterior, colisión, estrellamientos, pérdida de carril, pérdida de pista, volcamientos, entre otros), en la que en la mayoría de los casos las personas involucradas mueren (22.179) o simplemente quedan con algún tipo de lesión o discapacidad (19.999) que generalmente origina un daño irreversible a la víctima así como a las personas que los rodean. Esta situación, desencadena un importante problema social, por los daños tanto físicos como psicológicos que soportan estas personas (Organización Panamericana de la Salud, 2016).

En este mismo orden, las causas que registran cifras importantes de accidentes son: conducir desatento a las condiciones de tránsito (celular, pantallas de video, comida, maquillaje o cualquier otro elemento distractor) con un total de 4.989 casos que representa el 20.3\% del total; conducir el vehículo superando los límites máximos de velocidad con un total de 4.006 casos, que representa el 16.3\% del total; no respetar las señales reglamentarias de tránsito (pare, ceda el paso, luz roja en semáforo, entre otros) con un total de 2.766 casos, que representa el $11.2 \%$ del total; no guardar la distancia lateral mínima de seguridad entre vehículos con un total de 2.541 casos que representa el $10.3 \%$ del total; no mantener la distancia prudencial con respecto al vehículo que le antecede con un total de 2.301 casos que representa el $9.4 \%$ del total y conducir bajo la influencia de alcohol, sustancias estupefacientes o psicotrópicas con un total de 1.755 casos que representa el $7.1 \%$ de total (Agencia Nacional de Tránsito, 2019). Como es evidente en la mayoría de los casos la imprudencia del conductor es la principal causa para que se registren este tipo de acontecimientos.

A lo largo de la historia, la sociedad presentaba grandes inconvenientes en materia de solución de conflictos jurídicos en torno a eventos ocasionados por siniestros automovilísticos, a fin de determinar si una persona actuó o no con intención de causar un hecho determinado. Esto debido a que en un principio y hasta la actualidad, aquellas acciones realizadas con intención son castigados con mayor severidad; mientras que los actos realizados sin intención son castigados con menor severidad. En este sentido, dentro del contexto de la investigación, el dolo eventual surge por la necesidad de juzgar los hechos producidos por accidentes de tránsito, entendiéndose que el mismo se da cuando el sujeto no quiere el resultado típico; pero lo acepta, lo consiente, o carga con él. 
El dolo eventual constituye una nueva figura dentro del derecho penal, por esto hay que recordar las formas de dolo para entender su verdadera intención. En este sentido, el dolo tradicionalmente ha sido definido como la simbiosis de un conocer y un querer, que se ubica en la vertiente interna del sujeto, en su universo mental. En materia penal se dice que actúa dolosamente quien sabe que su acción es objetivamente típica y quiere su realización. A partir de esta definición, el dolo puede integrar dos importantes elementos: uno intelectual o cognitivo, que exige tener conocimiento o conciencia de los elementos objetivos del tipo penal respectivo; el otro elemento es volitivo, este implica querer realizarlos. No obstante, cada uno de estos componentes no siempre presentan los mismos grados de determinación ni de intensidad. Por esta razón, ha dado lugar a que la doctrina dominante distinga, en atención a la fluctuación de estos aspectos, tres clases de dolo: el directo de primer grado, el directo de segundo grado y el eventual (Velásquez y Wolffhügel, 2012).

En este sentido, el dolo directo de primer grado se entiende cuando el sujeto quiere el resultado típico. El dolo directo de segundo grado, conocido también de consecuencias necesarias, sucede cuando el sujeto no quiere el resultado típico; pero su producción se representa como cierta o segura. Y finalmente, el dolo eventual cuando el sujeto no quiere el resultado típico; sin embargo, lo acepta, lo consiente o carga con él, pese habérsele representado como posible o probable (Soto, 2015).

En este contexto, la gran mayoría de los accidentes de tránsito son producto de la excesiva velocidad, al sobrepasar los límites permitidos por la correspondiente Ley y su Reglamento, también debido al consumo de sustancias psicotrópicas o bebidas alcohólicas. Sin embargo, resulta ilógico presumir que cuando la persona conduce a excesiva velocidad y ocasionare la muerte de otra en un accidente de tránsito, haya tenido la intención de querer hacerlo, o producir tal efecto. No obstante, es de suma importancia esclarecer el hecho de que cuando la persona no ha querido ocasionar la muerte a otro; pero producto de su imprudencia, negligencia o impericia la ha causado, se está ante la presencia de un homicidio de tipo culposo por lo que, el causante de ello merece ser condenado por aquella acción producida.

En tal virtud, dentro del código ecuatoriano, el dolo eventual aún no se encuentra tipificado, dado que constituye una teoría nueva que existe en legislaciones de otras latitudes, así, al observar los resultados positivos que 
trae consigo su aplicación, es menester su incorporación inmediata dentro del Código Orgánico Integral Penal (2014), con la finalidad de disminuir los altos índices de accidentes de tránsito.

En este contexto, el objetivo del presente manuscrito se centra en realizar una revisión bibliográfica sobre aquellos aspectos del dolo eventual y la conducción temeraria de automotores. Para esto, se empleó la investigación dogmático-jurídica que se inserta en el enfoque cualitativo; puesto que se hizo una revisión del marco jurídico nacional e internacional referente al tema planteado para determinar conceptos, procedimientos y normativas vigentes dentro del contexto en el cual se desenvuelve. Asimismo, el tipo de investigación fue descriptiva con un diseño bibliográfico-documental, toda vez que permitió la interpretación de la información recopilada a través de la criticidad inferencial.

\section{Desarrollo}

Dentro de este apartado se presenta una revisión de conceptos, teorías, leyes y reglamentos en torno a las variables en estudio. En tal sentido, en la primera parte del documento, se encontrará información referente al dolo eventual analizando conceptos y teorías desde el punto de vista bibliográfico, mientras que desde un enfoque legal se presentan leyes o reglamentos que abordan esta temática. Posterior a esto, en la segunda parte, se realiza un acercamiento a lo que es la conducción temeraria de igual forma desde un enfoque teórico y legal. En virtud de lo mencionado, se presenta el desarrollo del mismo:

\section{Dolo eventual}

Resulta necesario previamente analizar las diferentes teorías que intentan dar una explicación al dolo eventual. Los esfuerzos doctrinarios se dirigieron hacia dos direcciones: cuál es el límite del dolo y de qué modo se fundamenta su aparición en la modalidad eventual (Pérez, 2017). Bajo este contexto, a continuación, dentro de la tabla 1 se describen las diferentes teorías que se han realizado con el objetivo de explicar el dolo eventual: 
Tabla 1. Teorías del dolo eventual

\begin{tabular}{|l|l|}
\hline Teorías & Descripción \\
\hline $\begin{array}{l}\text { Teoría del } \\
\text { consentimiento }\end{array}$ & $\begin{array}{l}\text { Teniendo como base que el dolo se configura con dos aspectos } \\
\text { (cognoscitivo y volitivo), entre el resultado y el accionar debe haber } \\
\text { una relación de voluntad. }\end{array}$ \\
\hline $\begin{array}{l}\text { La teoría } \\
\text { positiva del } \\
\text { consentimiento }\end{array}$ & $\begin{array}{l}\text { El despliegue doloso se presenta cuando hay una resignación al } \\
\text { resultado a pesar de que no lo quiera o lo desee. }\end{array}$ \\
\hline $\begin{array}{l}\text { La teoría de la } \\
\text { representación }\end{array}$ & $\begin{array}{l}\text { Sostiene que la representación de la posibilidad debería ser un } \\
\text { motivo contundente para dejar de actuar, y que la confianza en la no } \\
\text { producción del acto típico reúne la negación de la posibilidad de que } \\
\text { ocurra. }\end{array}$ \\
\hline $\begin{array}{l}\text { La teoría de la } \\
\text { indiferencia }\end{array}$ & $\begin{array}{l}\text { Internamente, el sujeto aprueba (o es indiferente) a las posibles } \\
\text { consecuencias negativas actuando con dolo, en cambio, cuando dichas } \\
\text { consecuencias las evalúa como indeseables, su comportamiento sería } \\
\text { culposo. Esta doctrina no hace una lectura satisfactoria, ya que lo } \\
\text { trascendental debe ser la decisión y no los deseos. }\end{array}$ \\
\hline $\begin{array}{l}\text { Las teorías } \\
\text { eclécticas }\end{array}$ & $\begin{array}{l}\text { Son una combinación de la teoría de representación y la del } \\
\text { conocimiento. En el aspecto cognoscitivo se requiere conocer la } \\
\text { peligrosidad de la conducta y en el volitivo que uno se resigne, } \\
\text { acepte, se conforme o lo asuma. }\end{array}$ \\
\hline $\begin{array}{l}\text { La teoría de la } \\
\text { probabilidad }\end{array}$ & $\begin{array}{l}\text { El requisito del dolo sería que la probabilidad sea más que la mera } \\
\text { posibilidad, pero menor que la probabilidad predominante. }\end{array}$ \\
\hline $\begin{array}{l}\text { La teoría de la } \\
\text { evitabilidad del } \\
\text { resultado }\end{array}$ & $\begin{array}{l}\text { Cuando el sujeto se ha representado la posibilidad del resultado, } \\
\text { pretende negar el dolo eventual solo cuando la voluntad conductora } \\
\text { del sujeto estuviera dirigida a la evitación del resultado. }\end{array}$ \\
\hline $\begin{array}{l}\text { Teoría de } \\
\text { Jakobs }\end{array}$ & $\begin{array}{l}\text { Hay dolo eventual cuando el sujeto evalúa la comisión del tipo como } \\
\text { producto de su accionar no siendo improbable su producción. }\end{array}$ \\
\hline Teoría de Roxin & $\begin{array}{l}\text { Existe certeza de la realización de la conducta típica y, sin embargo, } \\
\text { continúa con su accionar, resignándose (aceptando) a la eventual } \\
\text { realización del delito. }\end{array}$ \\
\hline
\end{tabular}

Fuente: Adaptado de Pérez (2017, p. 217-218) "El dolo eventual y la culpa consciente en accidentes automovilísticos".

El dolo eventual tuvo su origen a partir de la política criminal, como respuesta a las conductas diversas a la que la penalidad del delito culposo quedaba disminuida con base en la conducta desplegada, lo que hacía imposible el cumplimiento de la prevención general positiva de la pena, al manifestarse como una alternativa de solución más justa (Pérez, 2017).

Dentro de la legislación ecuatoriana no existe ninguna ley o norma que haga referencia al tratamiento del dolo eventual, lo único que se puede encontrar dentro de la actual legislación y específicamente en el Código Orgánico Integral 
Penal es la regulación del dolo, dado que dentro del artículo 26 se expone "Actúa con dolo la persona que tiene el designio de causar daño" (Código Orgánico Integral Penal, 2014, artículo 26, p. 10). Esta definición resulta ser la clásica pues no ha sufrido variación alguna con las reformas presentadas a lo largo de la historia.

El dolo eventual constituye la forma más débil de dolo, ya que en estos supuestos; tanto el elemento volitivo como el cognoscitivo aparecen con menor intensidad. El objetivo del sujeto que actúa con dolo eventual no es ocasionar el resultado; no obstante reconoce la posibilidad de que éste se produzca y, sin embargo, continúa actuando (Galarza, 2018). En este sentido, el responsable del dolo eventual no espera un resultado contrario a derecho; por ejemplo, una persona desea llegar sin ninguna novedad hasta su domicilio manejando su vehículo, para ello ejecuta maniobras peligrosas no permitidas. No obstante, este espera que no acontezca nada raro, o si en determinado momento llegare a pasar algo, confía en que pueda controlar el hecho en el instante en el que pueda perder el control de la situación (Ponce, 2016). De esta manera, el dolo eventual tiene su castigo, debido a que el autor tiene conocimiento previo de que su conducta presenta un riesgo y este puede provocar un resultado prohibido con consecuencias nefastas no deseadas por el autor.

Asimismo, desde otra perspectiva "el dolo eventual radica en el accionar con conocimiento por parte del sujeto de que su conducta ocasiona un peligro de actuación del tipo, es decir, el autor atribuye a su conducta la capacidad de realizar el tipo y no obstante no desiste de ella” (Parrado y Acevedo, 2013, p. 56). Dentro del dolo eventual, el infractor no tiene como finalidad de su accionar la ejecución del resultado delictivo, quizás ni lo desea y hasta cierto punto lo repudie.

Finalmente, de acuerdo con Elmelaj (2014) se configura el escenario del dolo eventual al momento que la intención se dirige indiferentemente a varios resultados, de manera que es como una ratificación anticipada a cualquiera de ellos que se resiste. De hecho, esto es un resultado característico en los delitos de impulso en el que "el sujeto no ha visualizado con claridad la relación entre la conducta y el resultado” (Piva, 2019, p. 138). El dolo eventual se caracteriza por la actitud que pone de manifiesto el sujeto, al asumir un riesgo que podría llegar a convertirse en daño o lesión. 


\section{Elementos que caracterizan el dolo eventual}

En el dolo eventual, el sujeto encamina su conducta hacia una finalidad de indolencia total para el Derecho Penal. Sin embargo, de este accionar se puede derivar un resultado típico que necesariamente hay que considerar como posible, sin que aquella circunstancia incida en la modificación del comportamiento del individuo. Es decir, continúa con su accionar y asume el riesgo de dicho acto. Asimismo, resulta evidente que luego de todas las consideraciones existen ciertos elementos representativos del dolo eventual. A modo de síntesis en la figura 1, se presentan los siguientes:

Figura 1. Elementos que caracterizan el dolo eventual

La acción supone un peligro para el bien jurídico protegido, y el sujeto reconoce ese peligro;

El sujeto crea un peligro jurídicamente desaprobado con la producción de un resultado que hubiera podido evitarse;

Representación de las consecuencias, del hecho o previstas como posibles, a través del conocimiento del hombre;

Se prevé como posible la producción del resultado, y se consiente, al continuar con la acción;

Indiferencia y menosprecio a las normas y reglamentos, y peor aún a la vida humana;

Continuar con el riesgo a pesar de la representación. Con este elemento el autor asume y consiente las consecuencias de la situación que creó;

No existe intención directa ni indirecta del delito, pero le resulta indiferente el resultado con consecuencia de su acción delictiva.

Fuente: Adaptado de Saigua (2014, p. 46).

\section{Conducción temeraria}

Para que exista una conducción temeraria, según Moreira y Guatemala (2015) el conductor debe efectuar al menos una de las siguientes conductas:

1. Realizar o participar en competencias de velocidad ilegales:

2. Sobrepasar los límites y rangos descritos en el Art. 191 del Reglamento General para la Aplicación de la Ley de Tránsito y Seguridad Vial siendo estos los siguientes: 
Tabla 2. Para vehículos livianos, motocicletas y similares

\begin{tabular}{|c|c|c|c|}
\hline Tipo de vía & Límite máximo & Rango moderado & $\begin{array}{c}\text { Fuera del rango } \\
\text { moderado }\end{array}$ \\
\hline Urbana & $50 \mathrm{~km} / \mathrm{h}$ & $50 \mathrm{~km} / \mathrm{h}-60 \mathrm{~km} / \mathrm{h}$ & $60 \mathrm{~km} / \mathrm{h}$ \\
\hline Perimetral & $90 \mathrm{~km} / \mathrm{h}$ & $90 \mathrm{~km} / \mathrm{h}-120 \mathrm{~km} / \mathrm{h}$ & $120 \mathrm{~km} / \mathrm{h}$ \\
\hline Rectas & $100 \mathrm{~km} / \mathrm{h}$ & $100 \mathrm{~km} / \mathrm{h}-135 \mathrm{~km} / \mathrm{h}$ & $135 \mathrm{~km} / \mathrm{h}$ \\
\hline Curvas & $60 \mathrm{~km} / \mathrm{h}$ & $60 \mathrm{~km} / \mathrm{h}-75 \mathrm{~km} / \mathrm{h}$ & $75 \mathrm{~km} / \mathrm{h}$ \\
\hline
\end{tabular}

Fuente: Adaptado del Reglamento General para la aplicación de la Ley Orgánica de Transporte Terrestre, Tránsito y Seguridad Vial (2012).

Tabla 3. Para vehículos de transporte público de pasajeros

\begin{tabular}{|c|c|c|c|}
\hline Tipo de vía & Límite máximo & Rango moderado & $\begin{array}{c}\text { Fuera del rango } \\
\text { moderado }\end{array}$ \\
\hline Urbana & $40 \mathrm{~km} / \mathrm{h}$ & $40 \mathrm{~km} / \mathrm{h}-50 \mathrm{~km} / \mathrm{h}$ & $50 \mathrm{~km} / \mathrm{h}$ \\
\hline Perimetral & $70 \mathrm{~km} / \mathrm{h}$ & $70 \mathrm{~km} / \mathrm{h}-100 \mathrm{~km} / \mathrm{h}$ & $100 \mathrm{~km} / \mathrm{h}$ \\
\hline Rectas & $90 \mathrm{~km} / \mathrm{h}$ & $90 \mathrm{~km} / \mathrm{h}-115 \mathrm{~km} / \mathrm{h}$ & $115 \mathrm{~km} / \mathrm{h}$ \\
\hline Curvas & $50 \mathrm{~km} / \mathrm{h}$ & $50 \mathrm{~km} / \mathrm{h}-65 \mathrm{~km} / \mathrm{h}$ & $65 \mathrm{~km} / \mathrm{h}$ \\
\hline
\end{tabular}

Fuente: Adaptado del Reglamento General para la aplicación de la Ley Orgánica de Transporte Terrestre, Tránsito y Seguridad Vial (2012).

Tabla 4. Para vehículos de transporte de carga

\begin{tabular}{|c|c|c|c|}
\hline Tipo de vía & Límite máximo & Rango moderado & $\begin{array}{c}\text { Fuera del rango } \\
\text { moderado }\end{array}$ \\
\hline Urbana & $40 \mathrm{~km} / \mathrm{h}$ & $40 \mathrm{~km} / \mathrm{h}-50 \mathrm{~km} / \mathrm{h}$ & $50 \mathrm{~km} / \mathrm{h}$ \\
\hline Perimetral & $70 \mathrm{~km} / \mathrm{h}$ & $70 \mathrm{~km} / \mathrm{h}-95 \mathrm{~km} / \mathrm{h}$ & $95 \mathrm{~km} / \mathrm{h}$ \\
\hline Rectas & $70 \mathrm{~km} / \mathrm{h}$ & $70 \mathrm{~km} / \mathrm{h}-100 \mathrm{~km} / \mathrm{h}$ & $100 \mathrm{~km} / \mathrm{h}$ \\
\hline Curvas & $40 \mathrm{~km} / \mathrm{h}$ & $40 \mathrm{~km} / \mathrm{h}-60 \mathrm{~km} / \mathrm{h}$ & $60 \mathrm{~km} / \mathrm{h}$ \\
\hline
\end{tabular}

Fuente: Adaptado del Reglamento General para la aplicación de la Ley Orgánica de Transporte Terrestre, Tránsito y Seguridad Vial (2012).

Las señales de tránsito deberán indicar tanto el límite de velocidad máximo como los rangos moderados. En caso de discrepancia entre los límites y rangos aquí indicados y los que se establezcan en las señales de tránsito, prevalecerán estas últimas. 
La Agencia Nacional de Tránsito y los Gobiernos Autónomos Descentralizados de ser el caso y manteniendo la debida coordinación, podrán establecer límites menores de velocidad, por razones de prevención y seguridad, así por ejemplo para el transporte escolar, o, en áreas de seguridad o carga, o limitar el acceso a determinadas vías respecto de determinado tipo de vehículos (Reglamento General para la Aplicación de la Ley de Transporte Terrestre Tránsito y Seguridad Vial, 2012, p. 42).

3. Conducir a una velocidad superior al treinta por ciento del límite de velocidad establecido en las vías urbanas y carreteras;

4. Aventajar en pendientes, curvas o puentes de forma indolente;

5. Realizar maniobras acrobáticas con el vehículo en la vía pública;

6. Cualquier otra conducta que constituya infracción a las normas de transito establecidas en el código respectivo, que sea ejecutada por los conductores con manifiesto desprecio por la vida, la integridad física de las personas, sus bienes o con notoria y deliberada transgresión a las normas de tránsito (Moreira y Guatemala, 2015, p. 5).

En este sentido cualquier conducta que realice el conductor sea en vías de primero, segundo y hasta tercer orden, es un condicionante para catalogar a la acción dentro del parámetro de la conducción temeraria. Lo que recae en una infracción de tránsito que es penalizada de acuerdo con las normas estipuladas dentro del órgano rector.

\section{Factores que conllevan a la conducción temeraria}

De acuerdo con Rauda y Pineda (2008), se identifican tres factores principales que conllevan a la conducción temeraria. En este sentido, a continuación, dentro de la figura 2 se presentan dichos factores:

Figura 2. Factores (conducción temeraria)



Fuente: Adaptado de Rauda y Pineda (2008, p. 10-12) 


\subsection{Conducción bajo estado de ebriedad}

Generalmente constituye una manera particular de intoxicación aguda por el alcohol, cuyo umbral de inicio es diferente, de acuerdo con los individuos se visualizan en un cuadro clínico conformado por ataxia total o parcial, sensorial, motriz y psíquica (Guanche, Martínez y Gutiérrez, 2014).

Las acciones generadas directamente por las personas que se encuentran en estado de ebriedad se producen con mayor frecuencia durante feriados o fines de semana. Por tanto, los individuos que se encuentran bajo los efectos del alcohol originan una agudeza en el cerebro, esta ocasiona comúnmente que el conductor pierda la capacidad de conducir su vehículo y pueda producir accidentes en la carretera con consecuencias muy fatales, como la muerte y lesiones del mismo individuo y de otras personas que nada tienen que ver con su entorno (Rivero, 2017).

En este sentido, en el Código Orgánico Integral Penal (2014, p. 58) dentro del capítulo octavo "infracciones de tránsito", en la sección segunda delitos culposos de tránsito en relación con la conducción bajo estado de embriaguez establece:

Artículo 385.- Conducción de vehículo en estado de embriaguez. - La persona que conduzca un vehículo en estado de embriaguez, será sancionada de acuerdo con la siguiente escala:

1. Si el nivel de alcohol por litro de sangre es de 0.3 a 0.8 gramos, se aplicará multa de un salario básico unificado del trabajador en general, pérdida de cinco puntos en su licencia de conducir y cinco días de privación de libertad. 2. Si el nivel de alcohol por litro de sangre es mayor de 0.8 hasta 1,2 gramos, se aplicará multa de dos salarios básicos unificados del trabajador en general, pérdida de diez puntos en su licencia de conducir y quince días de privación de libertad.

3. Si el nivel de alcohol por litro de sangre supera 1.2 gramos, se aplicará multa de tres salarios básicos unificados del trabajador en general, la suspensión de la licencia por sesenta días y treinta días de privación de libertad.

Para las o los conductores de vehículos de transporte público liviano o pesado, comercial o de carga, la tolerancia al consumo de cualquier sustancia estupefaciente, psicotrópica o preparado que las contengan es cero, y un nivel máximo de alcohol de 0,1 gramos por cada litro de sangre. En caso de exceder dicho limite, la sanción para el responsable será pérdida 
de treinta puntos en su licencia de conducir y pena privativa de libertad de noventa días.

Además, en todos estos casos, como medida preventiva se aprehenderá el vehículo por veinticuatro horas.

Asimismo, en el caso que producto de esta acción se registren muertes o lesiones causadas por accidentes de tránsito dentro de los artículos 376 y 379 del Código Orgánico Integral Penal (2014, p. 57) se establece:

Artículo 376.- Muerte causada por conductor en estado de embriaguez o bajo los efectos de sustancias estupefacientes, psicotrópicas o preparados que las contengan.- La persona que conduzca un vehículo a motor en estado de embriaguez o bajo los efectos de sustancias estupefacientes, psicotrópicas o preparados que las contengan y ocasione un accidente de tránsito del que resulten muertas una o más personas, será sancionada con pena privativa de libertad de diez a doce años, revocatoria definitiva de la licencia para conducir vehículos.

En el caso del transporte público, además de la sanción prevista en el inciso anterior, el propietario del vehículo y la operadora de transporte serán solidariamente responsables por los daños civiles, sin perjuicio de las acciones administrativas que sean ejecutadas por parte del organismo de transporte competente sobre la operadora.

Artículo 379.- Lesiones causadas por accidente de tránsito. - En los delitos de tránsito que tengan como resultado lesiones a las personas, se aplicarán las sanciones previstas en el artículo 152 reducidas en un cuarto de la pena mínima prevista en cada caso.

Serán sancionadas además con reducción de diez puntos en su licencia.

En los delitos de tránsito que tengan como resultado lesiones, si la persona conduce el vehículo en estado de embriaguez o bajo los efectos de sustancias estupefacientes, psicotrópicas o preparados que las contengan, se aplicarán las sanciones máximas previstas en el artículo 152, incrementadas en un tercio y la suspensión de la licencia de conducir por un tiempo igual a la mitad de la pena privativa de libertad prevista en cada caso.

\subsection{Conducción bajo efectos de las drogas}

Otros de los factores que conlleva al incremento desmesurado de la conducción temeraria es el consumo de sustancias psicotrópicas. Dado que, las drogas constituyen un estimulante que bloquea el cerebro con la realidad 
que se vive. De esta manera, entre las drogas con mayor frecuencia de uso se encuentran los alucinógenos, anfetaminas, barbitúricos, tranquilizantes, hipnóticos, derivados de la cocaína, entre otros (Cascales, 2017).

La utilización de sustancias estupefacientes bloquea la capacidad cerebral del conductor que ocasiona un descuido en el conductor a la hora de conducir. Dado que producen una serie de alteraciones en la conducción, lo que constituye un problema serio, desde el punto de vista de la seguridad en el tránsito.

Bajo este contexto, en relación con este aspecto, el Código Orgánico Integral Penal (2014, p. 58) dentro del capítulo octavo infracciones de tránsito, en la sección segunda: delitos culposos de tránsito en relación con la conducción bajo efectos de sustancias estupefacientes o psicotrópicas, establece:

Artículo 384.- Conducción de vehículo bajo efecto de sustancias estupefacientes, psicotrópicas o preparados que las contengan. - La persona que conduzca un vehículo bajo los efectos de sustancias estupefacientes, psicotrópicas o preparados que las contengan, será sancionada con reducción de quince puntos de su licencia de conducir y treinta días de privación de libertad: además como medida preventiva se aprehenderá el vehículo por veinticuatro horas.

Ahora bien, si producto de esta acción se registra un accidente con muerte, el Código Orgánico Integral Penal (2014, p. 57) dentro del artículo 376 establece:

Artículo 376.- Muerte causada por conductor en estado de embriaguez o bajo los efectos de sustancias estupefacientes, psicotrópicas o preparados que las contengan.- La persona que conduzca un vehículo a motor en estado de embriaguez o bajo los efectos de sustancias estupefacientes, psicotrópicas o preparados que las contengan y ocasione un accidente de tránsito del que resulten muertas una o más personas, será sancionada con pena privativa de libertad de diez a doce años, revocatoria definitiva de la licencia para conducir vehículos.

En el caso del transporte público, además de la sanción prevista en el inciso anterior, el propietario del vehículo y la operadora de transporte serán solidariamente responsables por los daños civiles, sin perjuicio de las acciones administrativas que sean ejecutadas por parte del organismo de transporte competente sobre la operadora. 


\subsection{Conducción transgrediendo las normas de seguridad vial}

La transgresión de las normas de seguridad constituyen el tercer elemento qué conlleva a la conducción temeraria, esta acción consiste en quebrantar las disposiciones legales comprendidas en la Ley General de Transporte Terrestre, Tránsito y Seguridad vial, Reglamento General de Tránsito y otras que regulan la forma de conducir (Rauda y Pineda, 2008).

En este sentido, en las carreteras a diario se puede observar el cometimiento de una serie de infracciones que, por simple que parezcan, recaen dentro del ámbito de la conducción temeraria que podría ocasionar consecuencias fatales, tanto para la persona que lo comete como para aquellos que no tienen nada que ver con aquel acto.

\section{Conclusiones}

Una vez concluido con el estudio quedan en evidencia las falencias existentes dentro del marco legal del país tanto con la Ley Orgánica de Transporte Terrestre y Seguridad Vial (2014) y el Código Orgánico Integral Penal (2014); dado que en ninguna de estas dos normativas existe algún artículo que castigue la conducción temeraria por conducir a exceso de velocidad, estado de embriaguez o bajo el efecto de algún tipo de sustancia psicotrópica fundamentado en el dolo eventual. Por tanto, resulta necesario que dentro de nuestra legislación cada vez se contemplen nuevas modalidades de delitos, debido a que las circunstancias son cambiantes al igual que la sociedad.

Por otro lado, el dolo eventual constituye una de las modalidades de delitos contempladas en otras legislaciones. Sin embargo, dentro de la nuestra aún no se encuentra tipificada como tal dentro del Código Orgánico Integral Penal, pues únicamente se halla una descripción general del dolo en el artículo 26 (Código Orgánico Integral Penal, 2014, artículo 26). Esta situación ha llamado enormemente la atención de diferentes juristas, dados los problemas sociales que en la actualidad causan los accidentes de tránsito.

Finalmente, la mayor parte de accidentes de tránsito se producen por causas, motivos o errores humanos; ya que la acción de manejar un vehículo o transitar por las vías, constituye una actividad con un alto grado de riesgo, por lo que al momento de conducir se requieren altos niveles de atención por parte de la persona que lo realiza. El conductor de un vehículo representa 
un procesador de información, debido a que recepta, decide y ejecuta una acción en tiempos muy cortos y continuos. Por lo tanto, es indispensable que sus funciones psicofísicas estén en coordinación y óptimas condiciones, para evitar cualquier tipo de percance en las vías. Los factores que ponen en peligro todo este proceso constituyen el exceso de velocidad, la ingestión de bebidas alcohólicas o el consumo de sustancias psicotrópicas, aumentando de forma significativa las probabilidades de desencadenar en un siniestro de tránsito.

\section{Referencias bibliográficas}

Agencia Nacional de Tránsito. (2019). Estadísticas sobre siniestros de tránsito. Website: https://www.ant.gob.ec/index.php/estadisticas.

Cascales, J. (2017). Delito de conducción bajo la influencia de alcohol, drogas tóxicas, estupefacientes o sustancias psicotrópicas (Tesis de pregrado, Universitas Miguel Hernández, Alicante, España).

Ecuador. Asamblea Nacional República de Ecuador. Reglamento a Ley de Transporte Terrestre Tránsito y Seguridad Viial (2012). Editora Nacional, Quito, pp, 91.

Ecuador. Asamblea Nacional República de Ecuador. Código Orgánico Integral Penal (2014). Editora Nacional, Quito, pp, 144.

Ecuador. Asamblea Nacional República de Ecuador. Ley Orgánica de Transporte Terrestre Tránsito y Seguridad Vial (2014). Editora Nacional, Quito, pp. 66.

Ecuador. Asamblea Nacional República de Ecuador. Reglamento General para la Aplicación de la Ley Orgánica de Transporte Terrestre, Tránsito y Seguridad Vial (2015). Editora Nacional, Quito, pp. 51.

Elmelaj, M. (2014). La frontera entre el dolo eventual y la imprudencia consciente (Tesis de maestría, Universidad de Sevilla, Mendoza, Argentina).

Galarza, J. (2018). El estudio dogmático y jurídico del Estado de necesidad y las causas de justificación en la teoría del delito (Tesis de pregrado, Universidad Técnica de Machala, Machala, Ecuador).

Guanche, H., Martínez, C. y Gutiérrez, F. (2014). Efecto del alcohol en la capacidad de conducción de vehículos automotores. Revista Cubana de Salud Pública, 33(1), pp. 1-6.

Moreira, J. y Guatemala, R. (2015). Determinar las causas de la conducción temeraria de los motociclistas, en la Rotomsa. El periodista en el primer trimestre del año 2015 (Tesis de maestría, Academia de Policia "Walter Mendoza Martínez", Managua, Nicaragua).

Organización Mundial de la Salud. (2014). Informe sobre la situación mundial de la seguridad vial. Organización Mundial de la Salud.

Organización Panamericana de la Salud. (2016). La seguridad vial en la región de las Américas. Organización Panamericana de la Salud.

Parrado, R. y Acevedo, Y. (2013). El límite entre el dolo eventual y la culpa con representación en los accidentes de tránsito a la luz de la ley 599 de 2000 en Colombia (Tesis de maestría, Universidad Libre, Bogotá, Colombia).

Pérez, C. A. (2017). El dolo eventual y la culpa consciente en los accidentes automovilísticos: la perspectiva desde el derecho penal argentino. Jurídicas Cuc, 13(1), pp. 213-232. 
Piva, G. E. (2019). Teoría del Delito y el Estado Social y Democrático de Derecho. Bosch Editor.

Ponce, G. (2016). El dolo, concepto limitado en el Código Orgánico Ontegral Penal. Dolo Directo, indirecto y eventual. Website: https://lexadvisorecuador.com/2019/08/14/ el-dolo-concepto-limitado-en-el-codigo-organico-integral-penal/

Rauda, N. y Pineda, J. (2008). La conducción temeraria de vehículos de motor en el Salvador (Teis de maestría, Uniersidad Tecnológica de El Salvador, San Salvador, El Salvador).

Rivero, D. (2017). El alcohol como factor decencadenate en las conductas delictivas viales (Tesis de maestría, Universidad del Aconcagua, Mendoza, Argentina).

Saigua, L. (2014). Proyecto de Ley Reformatoria que incremente el dolo eventual como modalidad de imputación en los delitos asociados a los de tránsito (Tesis de pregrado, Universidad Regional Autónoma de los Andes, Riobamba, Ecuador).

Soto, J. (2015). Dolo eventual: tratamiento en el Derecho Penal Venezolano (Tesis de maestría, Universidad de Carabobo, Valencia, Venezuela).

Velásquez, F. y Wolffhügel, C. (2012). La diferencia entre el dolo eventual y la culpa consciente en la reciente jurisprudencia. Cuadernos de Derecho Penal, (6), pp. 99-180. 